\title{
Graphene hydrogels with embedded metal nanoparticles as efficient catalysts in 4-nitrophenol reduction and methylene blue decolorization
}

\author{
Kamila Żelechowska*, Izabela Kondratowicz, Maria Gazda \\ Gdansk University of Technology, Faculty of Technical Physics and Applied Mathematics, Narutowicza 11/12, \\ 80-233 Gdansk, Poland \\ "Corresponding author: e-mail: kzelechowska@mif.pg.gda.pl
}

\begin{abstract}
Synthesis and characterization of the graphene hydrogels with three different metallic nanoparticles, that is $\mathrm{Au}, \mathrm{Ag}$ and $\mathrm{Cu}$, respectively is presented. Synthesized in a one-pot approach graphene hydrogels with embedded metallic nanoparticles were tested as heterogeneous catalysts in a model reaction of 4-nitrophenol reduction. The highest activity was obtained for graphene hydrogel with $\mathrm{Cu}$ nanoparticles and additional reaction of methylene blued degradation was evaluated using this system. The obtained outstanding catalytic activity arises from the synergistic effect of graphene and metallic nanoparticles. The hydrogel form of the catalyst benefits in the easiness in separation from the reaction mixture (for example using tweezers) and reusability.
\end{abstract}

Keywords: graphene hydrogel, metallic nanoparticles, heterogeneous catalyst, 4-nitrophenol reduction.

\section{INTRODUCTION}

Many of industrially important reactions i.e oil refining or bulk chemicals production require usage of catalysts. Heterogeneous catalysts are most frequently used, because of the easiness of their recovery and recycling and their conformity in continuous processing ${ }^{1}$. With the nanotechnology development, metallic nanoparticles gained much interest as potential catalysts in a number of reactions, including selective reduction of nitro compounds. Among others, gold nanoparticles (Au NPs) are the most frequently studied and immensity of papers can be found in the literature. The recent progress in state-of-the-art gold nanoparticle catalysts for chemoselective hydrogenations under liquid-phase conditions was reviewed in $^{2}$. Silver and copper nanoparticles were also examined as a cheaper alternative to Au NPs in the nitro arenes reduction, however the literature in this topic is limited ${ }^{3-5}$. Metallic nanoparticles can be used in a colloidal form or they can be supported on different solids, either inorganic or organic ${ }^{3-16}$. Graphene and its derivatives (as graphene oxide or reduced graphene oxide) found its place in the catalysis as a support for nanoparticles, showing in some cases synergistic effect. The large surface area, electrical conductivity and mechanical strength make graphene the most promising substrate to be coupled with NPs catalysts to achieve high catalytic performance. Adsorption of reactants on graphene planes enhances their reduction by transferring free electrons from electron-rich graphene surface. Secondly, using graphene as a platform for metallic nanoparticles immobilization minimizes the NPs agglomeration and ensures the high dispersity of the catalyst. The reduction of 4-nitrophenol (4NP) to 4-aminophenol (4AP) has been performed using graphene/nanoparticles composites based on metals (e.g. $\mathrm{Au}, \mathrm{Pt}, \mathrm{Pd}, \mathrm{Ag}, \mathrm{Ni}$ etc.) or alloys $\left(\mathrm{FeCo}, \mathrm{ZnNi}\right.$ ) with $\mathrm{NaBH}_{4}$ as the reducing agent ${ }^{11-17}$. The mentioned 4-nitrophenol/ $\mathrm{NaBH}_{4}$ system is the preferred model for studying the activity of catalysts because the reaction can be easily followed by UV-vis spectroscopy.

Recent studies have shown that the self-assembly of graphene oxide (GO) under proper conditions leads to the creation of free-standing reduced graphene oxide
(rGO) hydrogels. Since the pioneer work of Shi's group, the self-assembled graphene hydrogels have gained much attention in different fields of science, including catalysis ${ }^{18-20}$. Such graphene hydrogel, comprising reduced graphene oxide and water is an interesting candidate for NPs support. Graphene hydrogel-NPs nanocomposites can be made by growing NPs directly on rGO surfaces in one-pot approach or by the simple mixing of pre-made NPs with GO, followed by its reduction. Au/graphene hydrogel synthesis, characterization and its use for catalytic reduction of 4-nitrophenol was described by Li et al. ${ }^{21}$ The Au/graphene hydrogel was prepared through a hydrothermal process with graphene oxide as the graphene precursor, chloroauric acid as $\mathrm{Au}$ precursor and triethylenetetramine as a reductant. The $\mathrm{Au}$ nanoparticles synthesized in that one-pot synthesis were uniformly supported on the interconnected graphene sheets. More complex approach of $\mathrm{Ag} / \mathrm{graphene}$ nanocomposite manufacturing was proposed by Dubey et al. ${ }^{22}$ Graphene oxide and carbon spheres doped with silver nanoparticles were prepared separately, to be in the next step converted into hydrogel with the usage of cysteine. Such hybrid structures were found to be an efficient catalyst for the reduction of 4-NP into 4-AP using $\mathrm{NaBH}_{4}$ as a reducing agent.

The Ag or Au 3D graphene-based porous monoliths were prepared by using an unidirectional freeze-drying method. The gold containing 3D porous monoliths showed superior catalytic activity for the reduction of 4-nitrophenol, while silver-based catalyst was tested in the Suzukie-Miyaura coupling reaction with unsatisfactory results ${ }^{23}$.

A simple one-step method for fabricating graphenebased hydrogels with interconnected 3D networks using $\mathrm{Cu}$ nanoparticles was developed. During this process, graphene oxide was reduced by $\mathrm{Cu}$ nanoparticles to form self-assembled graphene hydrogel with embedded nanoparticles. The unreacted $\mathrm{Cu}$ and $\mathrm{Cu}_{2} \mathrm{O}$ nanoparticles enwrapped in the graphene hydrogel structure showed good catalytic efficiency towards $4 \mathrm{NP}^{24}$. The usage of heterogeneous catalysts in a form of hydrogel composite is one of the most promising methodologies in developing green organic transformation processes because of 
their intrinsic advantages such as easiness in separation from the reaction mixture (for example using tweezers) and reusability. Three dimensional graphene/metal nanocomposites have also unique properties, obtained through the cooperative action between the active metal species and carbonaceous support, providing unusual and efficient catalytic routes for organic synthesis. Moreover, harmful and hazardous to our environment compounds, as for example nitroarenes or dyes can be removed from wastewater via. catalytic reduction in the presence of graphene composites.

Here we present the synthesis and characterization of the graphene hydrogels with three different metallic nanoparticles, that is $\mathrm{Au}, \mathrm{Ag}$ and $\mathrm{Cu}$, respectively. The catalytic performance of all hybrid structures was evaluated using model reaction of 4-nitrophenol reduction with the excess of $\mathrm{NaBH}_{4}$. The best results were obtained for graphene hydrogel containing $\mathrm{Cu}$ NPs, thus it was additionally tested towards decolorization of methylene blue solution.

\section{EXPERIMENTAL}

Graphite flakes, $\mathrm{AgNO}_{3}, \mathrm{HAuCl}_{4} \cdot \mathrm{xH}_{2} \mathrm{O}$ were purchased in Sigma-Aldrich. Potassium permanganate $\left(\mathrm{KMnO}_{4}\right), \mathrm{CuCl}_{2} \cdot 2 \mathrm{H}_{2} \mathrm{O}, \mathrm{L}$-ascorbic acid, $98 \%$ sulfuric acid $\left(\mathrm{H}_{2} \mathrm{SO}_{4}\right), 85 \%$ phosphoric acid $\left(\mathrm{H}_{3} \mathrm{PO}_{4}\right), 30 \%$ hydrogen peroxide $\left(\mathrm{H}_{2} \mathrm{O}_{2}\right), 35 \%$ hydrochloric acid $(\mathrm{HCl})$, and ethanol were purchased in POCh (Gliwice, Poland).

During the experiments the magnetic stirrer Heidolph MR hei-standard with hot plate was used and the centrifugation for all samples was done using Chemland model P3032 centrifuge with the speed $15000 \mathrm{rpm}$ for $10 \mathrm{~min}$.

UV-Vis spectra were measured using Lambda 10, Perkin Elmer.

The morphology of reduced graphene oxide hydrogels with NPs was observed with a scanning electron microscopy (ESEM Quanta Feg 250, FEI). The elemental analysis was performed by energy-dispersive X-ray (EDX) spectroscopy was performed using the EDAX Genesis APEX 2i with Apollo X SDD spectrometer at $10 \mathrm{kV}$.

The presence of crystalline phases was checked by X-ray diffraction with the use of a Philips X'Pert diffractmeter system. The XRD patterns of the samples were recorded at room temperature with $\mathrm{CuK}_{\alpha}$ radiation. Qualitative analysis of diffraction spectra was carried out with ICDD PDF database ${ }^{25}$.

Microphotographs were recorded using confocal microscope Olympus Lext 4000.

Raman spectra were recorded using Renishaw InVia spectroscope with argon ion laser operating at 514.5 $\mathrm{nm}$ focused through a 50x objective. Collected light was dispersed through a triple monochromator and detected with a charge-coupled device. The spectra were collected in the dark, with resolution of $2 \mathrm{~cm}^{-1}$ in the range of $100-3200 \mathrm{~cm}^{-1}$.

The FTIR spectra were recorded using $\mathrm{KBr}$ pellet method on Perkin Elmer Frontier spectrophotometer.

\section{Synthesis of graphene oxide}

Graphene oxide (GO) was prepared using method previously described by $\mathrm{us}^{\mathbf{2 6}}$. Shortly, $0.2 \mathrm{~g}$ of graphite powder was mixed with $0.6 \mathrm{~g}$ of $\mathrm{KMnO}_{4}$ and then the mixture of $\mathrm{H}_{2} \mathrm{SO}_{4} / \mathrm{H}_{3} \mathrm{PO}_{4}(12 \mathrm{ml}: 3 \mathrm{ml})$ was slowly added. The suspension was left for $24 \mathrm{~h}$ with the magnetic stirring. Then $3 \%$ of hydrogen peroxide was added in order to stop the reaction. The suspension was centrifuged at $15000 \mathrm{rpm}$ for $10 \mathrm{~min}$ and washed several times with deionized water, $3 \%$ of $\mathrm{HCl}$ and with water once again. It was dried under vacuum overnight. To obtain graphene oxide suspension $100 \mathrm{mg}$ of GO was dispersed in $100 \mathrm{ml}$ of deionized water using ultrasonic bath for $4 \mathrm{~h}$. The prepared $1 \mathrm{mg} / 1 \mathrm{ml}$ suspension was used in further experiments.

The graphite flakes in a certain stage of their transformation from graphite to GO were withdrawn from the reaction mixture with a pipette and sandwiched between a microscope slide and a glass coverslip.

\section{Synthesis of graphene hydrogel/NPs composites}

For the creation of graphene hydrogel, the hydrothermal method of reduction through self-assembly of reduced graphene oxide sheets was used for all samples. Ascorbic acid (AA) was chosen as a reductant of graphene oxide as well as metal ions to form nanoparticles. The previously prepared GO suspension $(8 \mathrm{ml}, 1 \mathrm{mg} / \mathrm{ml})$ was mixed in a vial with metal precursor, that is $\mathrm{HAuCl}_{4}, \mathrm{AgNO}_{3}$ or $\mathrm{CuCl}_{2}$ and was sonicated for 1 minute. The $\mathrm{HAuCl}_{4}$, $\mathrm{AgNO}_{3}$ or $\mathrm{CuCl}_{2}$ were weighted in such amounts to keep the same metal content in all hydrogels, that is $1: 1$ $\mathrm{w} / \mathrm{w}$ GO to metal. After addition of the excess of the reducing agent ( $32 \mathrm{mg} \mathrm{AA}$ ) the vials were sealed and heated up in an oil bath up to $90^{\circ} \mathrm{C}$. After 2-3 hours, the reduced graphene oxide hydrogels with embedded nanoparticles were formed. The created structures were thoroughly washed with deionized water in order to remove unreacted reductant and by-products. Purged hydrogels were stored in water at rt. To verify their catalytic activity the hydrogels were removed from the vials and put without drying into the reaction mixture.

Before microscopic and spectroscopic characterization the hydrogels should be dried, and therefore converted into aerogels. The drying protocol was as follows. Hydrogels were washed several times with deionized water and kept in ethanol for several days for the solvent exchange. All hydrogels were dried simultaneously under supercritical conditions for ethanol (63 bar, $\left.241^{\circ} \mathrm{C}\right)$. Teflon beakers containing hydrogels were placed in an autoclave with $100 \mathrm{ml}$ of ethanol. Ethanol was added to the beakers and to the body of the autoclave. Firstly, the autoclave was flushed a few times with $\mathrm{N}_{2}$ gas and pre-pressurized to 50 bar. Samples were heated up to $300^{\circ} \mathrm{C}$ with a heating rate of $5^{\circ} \mathrm{C} / \mathrm{min}$ and kept in this temperature for $2 \mathrm{~h}$. Then the autoclave was evacuated at a constant temperature with a rate of about $0.7 \mathrm{bar} /$ min. Finally, the autoclave was cooled down to room temperature. If necessary, the samples were disintegrated prior measurements.

\section{Study on the catalytic activity of GO/NPs hydrogels}

In order to investigate the catalytic activity of the as-prepared graphene-metallic hydrogels at room temperature, the reduction reaction of 4-nitrophenol by $\mathrm{NaBH}_{4}$ was carried out. The aqueous solutions of $4 \mathrm{NP}$ $\left(10^{-3} \mathrm{M}\right)$ and $\mathrm{NaBH}_{4}(0.1 \mathrm{M})$ were prepared directly before experiments. The $3 \mathrm{ml}$ of $4 \mathrm{NP}$ solution and 2 
$\mathrm{ml}$ of $\mathrm{NaBH}_{4}$ solution were added to the vial containing catalyst and the mixture was diluted to $15 \mathrm{ml}$.

The methylene blue aqueous solution $\left(5 \mathrm{ml}, 10^{-5} \mathrm{M}\right)$ was mixed with $\mathrm{NaBH}_{4}(10 \mu \mathrm{l}, 0.1 \mathrm{M})$. The UV-Vis spectra were recorded in the range of $200-900 \mathrm{~nm}$.

\section{RESULTS AND DISCUSSION}

Synthesis and characterization of graphene hydrogels.

The GO was successfully prepared by method previously described by us $^{\mathbf{2 6}}$. The material was characterized using Raman, UV-Vis and FTIR spectroscopy, and scanning electron microscopy. The details can be found in our previous papers ${ }^{26,27}$ and in the section below. Additionally, the progress of the oxidation of graphite during the synthesis was monitored by optical microscopy. In Figure 1. graphene flake at different stages of oxidation is presented. Figure 1A shows blue graphene flake at the preliminary stage of the reaction. The blue color is characteristic for intercalation product of graphite with sulfuric acid. In the next photos (Fig. 1B and B) not-fully and fully-oxidized graphite flakes can be seen. Oxidized areas along the flake's perimeter appear semitransparent light-brown, whereas the unoxidized areas in the center of the flake appear blue-colored (Fig. 1A). In the case of fully oxidized flakes they whole surface is semitransparent and brownish.

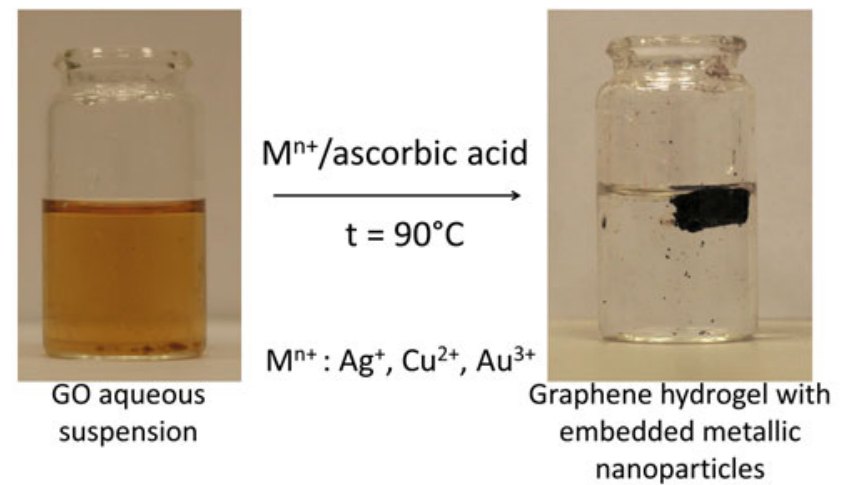

Figure 2. Scheme of hydrogel/NPs composites synthesis

and XRD spectroscopy. In Figure 3 the spectra recorded for $\mathrm{GO}$ and $\mathrm{rGO}$ can be seen. As for the spectroscopic measurements the dried hydrogel should be disintegrated, it was denoted in Figure 3 as rGO as it was not in the form of hydrogel. The presented results are focused only on changes concerning the carbonaceous structure. The structural and spectroscopic features connected with embedded nanoparticles will be discussed later.

Figure 3A shows FTIR spectrum of GO and rGO. Distinct band in GO spectrum at $1735 \mathrm{~cm}^{-1}$ confirms the presence of carbonyl groups. The small band at $1590 \mathrm{~cm}^{-1}$ is assigned to the stretching vibrations of $\mathrm{C}=\mathrm{C}$ conjugated with $\mathrm{C}=\mathrm{C}$ or $\mathrm{C}=\mathrm{O}$ bonds. The broad multiple band
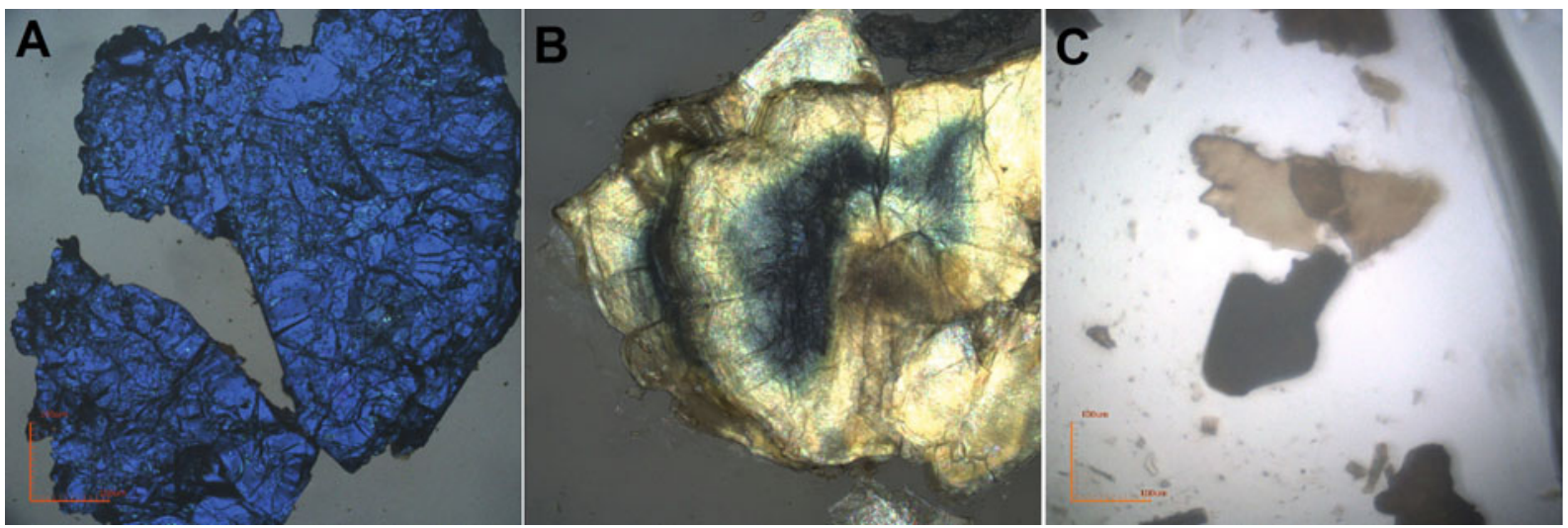

Figure 1. Optical images of graphite flakes at different stages of oxidation: A) intercalation product of graphite with sulfuric acid formed in the beginning of the reaction; B) partially oxidized and C) fully oxidized graphite

This simple and low-cost method can be used to control the reaction progress, instead of the expensive and not always available electron microscopy. Such approach can be included into the quality management in the scaled-up production of GO.

The graphene hydrogel formation was carried out by known hydrothermal method ${ }^{\mathbf{1 8}, 26,27}$. The cylindrically shaped hydrogel/NPs composites were obtained in all cases. The representative image, presenting hydrogel formation can be seen in Figure 2. The obtained hydrogels were denoted as $\mathrm{GH} \_\mathrm{Au}, \mathrm{GH} \_\mathrm{Ag}$ and $\mathrm{GH} \_\mathrm{Cu}$, for reduced graphene oxide hydrogel with gold, silver and copper nanoparticles, respectively.

After the reduction, the structural and chemical properties of GO change dramatically and different spectroscopic methods can be used to characterize GO and reduced $\mathrm{GO}$ ( $\mathrm{rGO}$ ). The conversion of $\mathrm{GO}$ into hydrogel was observed with naked eye and the changing properties were monitored by FTIR, UV-VIS, Raman between 1420 and $1000 \mathrm{~cm}^{-1}$ are placed in the region characteristic for stretching of $\mathrm{C}-\mathrm{O}$ bond in alcohols and epoxides. The broad, strong band centered at c.a. 3300 $\mathrm{cm}^{-1}$ and second strong band at $1640 \mathrm{~cm}^{-1}$ refer to the water molecules bonded to GO due to its hydrophilic nature. After reduction, all strong bands disappeared proving the successful removal of oxygen containing groups. The vibrational bands originating from water molecules are also not visible as hydrophobic nature of $\mathrm{rGO}$ do not facilitate the water adsorption. The UV-VIS spectra of $\mathrm{GO}$ and $\mathrm{rGO}$ aqueous suspensions are presented in Figure 3B. The main maximum absorption band at GO spectrum, observed at $230 \mathrm{~nm}$, can be ascribed to $\pi \rightarrow \pi^{*}$ electron transition in conjugated carbon-carbon double bonds in the GO plane. The second, smaller band at 310 $\mathrm{nm}$ is connected with $\mathrm{n} \rightarrow \pi^{*}$ transition of nonbonding electrons in oxygen atoms connected with double $\mathrm{C}=\mathrm{C}$ bonds. Upon reduction, a red shift of the first peak and the disappearance of the second one was observed 

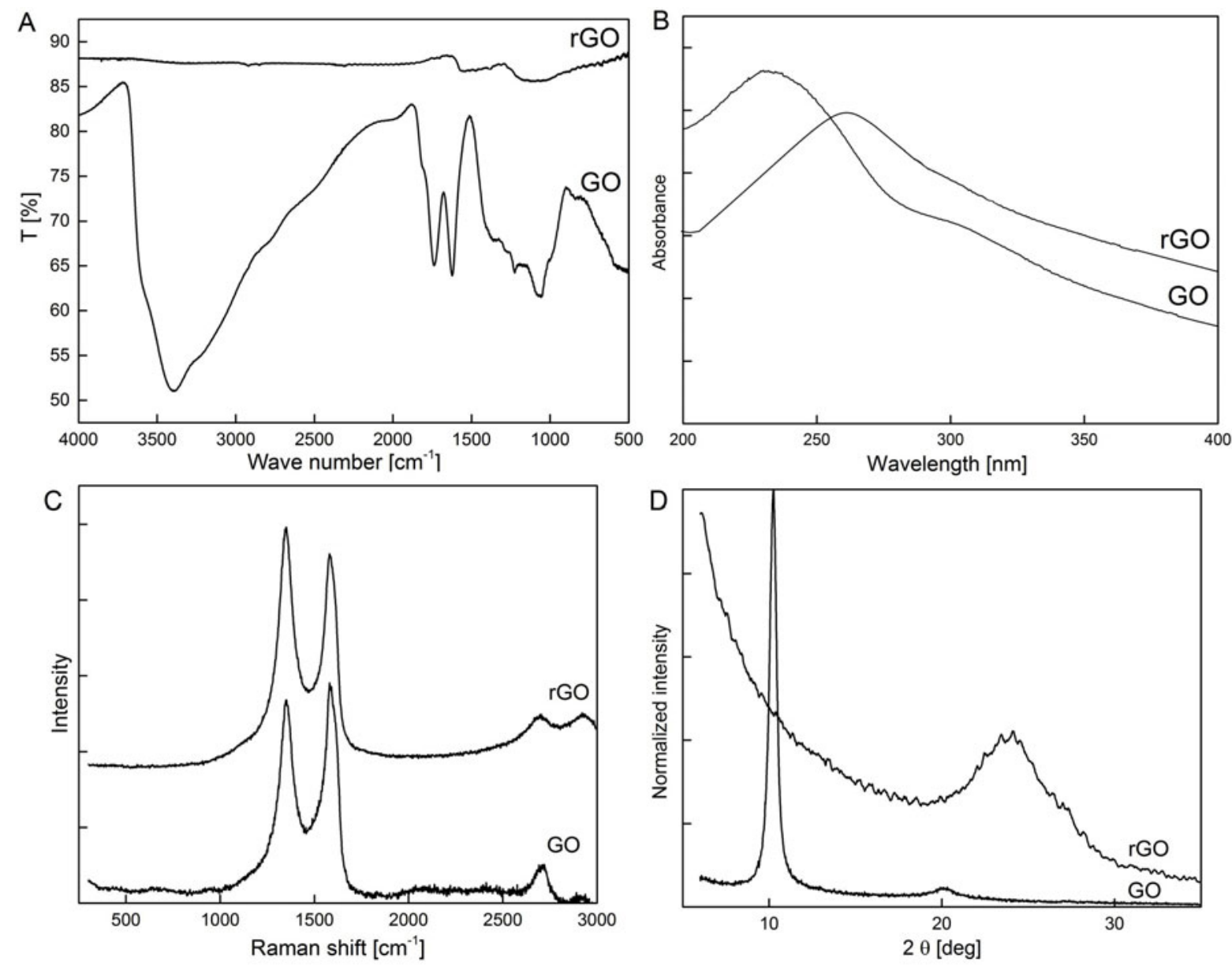

Figure 3. A) FTIR; B) UV-VIS; C) Raman and D) XRD spectra of GO and rGO

(Fig. 3 B, rGO), confirming again the successful removal of oxygen functionalities.

Raman spectroscopy is very frequently used to characterize carbonaceous materials, including GO and its derivatives. The Raman spectrum of GO (Fig. 3C, GO) consists of two main modes: a broad D mode at $1350 \mathrm{~cm}^{-1}$ and $\mathrm{G}$ mode at $1580 \mathrm{~cm}^{-1}$. The prominent $\mathrm{D}$ peak arises from the structural imperfections created by the attachment of hydroxyl and epoxide groups on the carbon basal plane and $G$ peak refers to the vibration of carbon-carbon bonds in a basal plane of GO. The overtone 2D-band can be seen in the range of 2700 and $3000 \mathrm{~cm}^{-1}$. The intensity of the overtone 2D-band with respect to the $D$ and $G$ peaks is small. Raman spectrum of rGO (Fig. 3C) is similar to the spectrum of its precursor, however showing higher $\mathrm{D}$ band intensity than $\mathrm{G}$ band. As reported elsewhere comparing the $\mathrm{D}$ to $G$ ratios do not offer any insights about the chemical changes in the samples, in contrast to the changes of $2 \mathrm{D}$ to $\mathrm{G}$ ratios ${ }^{28}$. Moreover, after the reduction a small peak shift for the 2D-band and no obvious shift for the D-band or the G-band can be observed, showing that changes in the overtone region of the Raman spectrum is reduction indicative. The small 2D-peak shift, together with decrease of its intensity in respect to $\mathrm{G}$ band $\left(\mathrm{I}_{2 \mathrm{D}} /\right.$ $\mathrm{I}_{\mathrm{G}}=0.12$ for $\mathrm{GO}$ and $\mathrm{I}_{2 \mathrm{D}} / \mathrm{I}_{\mathrm{G}}=0.05$ for $\mathrm{rGO}$ ) confirms the reduction of GO to rGO. The last method used to characterize the obtained materials was XRD spectroscopy. The XRD patterns of GO and rGO are shown in Figure 3D. The XRD pattern of GO reveals the diffraction peak at $2 \theta=10.23^{\circ}$ which can be attributed to the (002) diffraction line of graphite. The Bragg's equation was used to calculate the interlayer spacing (d-spacing) of $\mathrm{GO}$ which was equal to $0.863 \mathrm{~nm}$. The XRD pattern of rGO shows a diffraction peak at $2 \theta$ $=23.77^{\circ}$ which corresponds to the d-spacing $0.374 \mathrm{~nm}$. The decrease in the interlayer spacing is a proof of the chemical reduction and the removal of oxygen-containing functional groups from GO plane. The broad XRD signal of rGO indicated the poor ordering of sheets along their stacking direction. It also demonstrated that the structure of rGO was composed of the few-layer stacked sheets, which was consistent with the results obtained by SEM imaging.

In order to characterize the obtained hydrogel/NPs composites they were washed several times with deionized water and kept in ethanol for several days for the solvent exchange. All hydrogels were dried simultaneously under supercritical conditions for ethanol. Dried hydrogels were examined by scanning electron microscopy (SEM) with, UV-Vis spectroscopy and X-ray diffraction (XRD).

Due to the surface plasmon resonance phenomenon colloids of metallic nanoparticles can be characterized by UV-vis spectroscopy. The synthesized hydrogel/NPs composites were dispersed in water and absorption spectra were recorded. In Figure 4 the absorbance spectrum of three different samples can be seen. The characteristic bands at wavelengths ca. $440 \mathrm{~nm}, 530 \mathrm{~nm}$ and $570 \mathrm{~nm}$ refer to silver, gold and copper nanoparticles, respectively. The considerable broadening of the absorption band in the case of $\mathrm{GH} \mathrm{Au}$ and $\mathrm{GH} \mathrm{Ag}$ samples indicates the polydispersity of the analyzed nanoparticles, which is in 


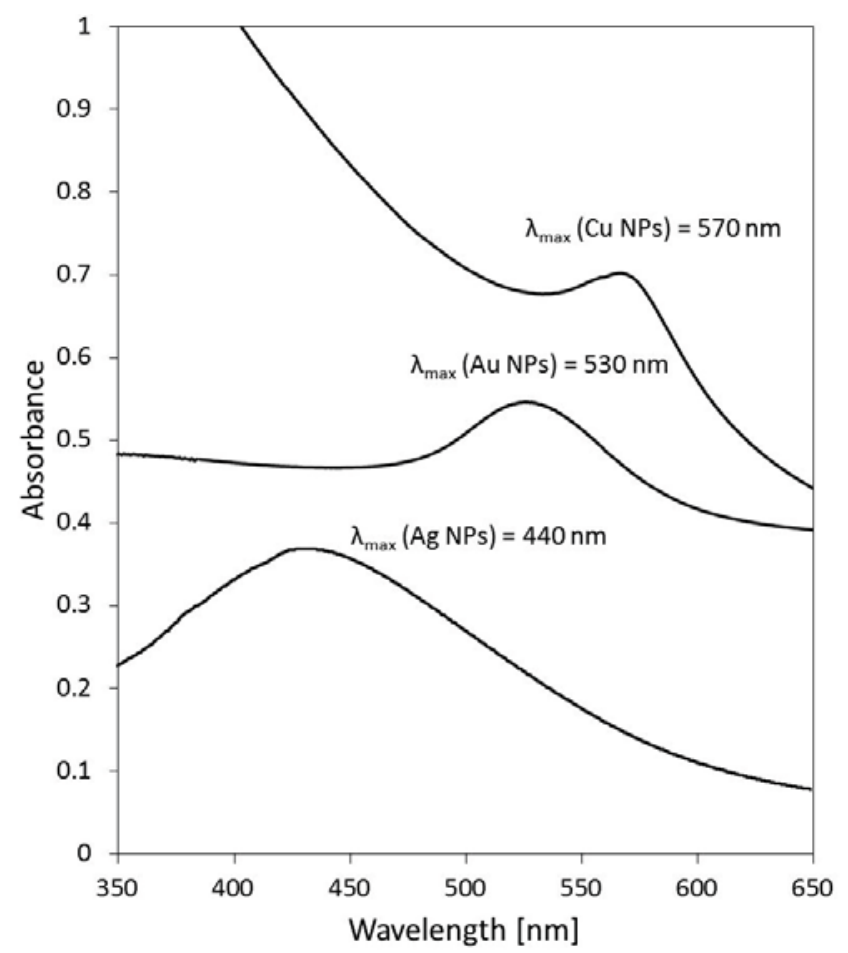

Figure 4. Absorption spectra of dispersed $\mathrm{GH}_{-} \mathrm{Au}, \mathrm{GH} \_\mathrm{Ag}$ and $\mathrm{GH}_{-} \mathrm{Cu}$, showing characteristic bands for $\mathrm{Au}$, $\mathrm{Ag}$ and $\overline{\mathrm{Cu}}$ nanoparticles

agreement with SEM images. The absorption spectrum of GH_Cu sample is in agreement with previously reported results for $\mathrm{Cu}$ nanoparticles of diameters below $10 \mathrm{~nm}^{29}$.

Additional examination of the samples was carried out by X-ray diffraction method (XRD). The obtained XRD patterns confirmed the presence of metallic nanoparticles in the reduced graphene matrix (Fig 5). In the case of $\mathrm{GH}$ Au sample the reflections at $2 \theta$ angle $38.3^{\circ}, 44.5^{\circ}$, $64.7^{\circ}$ and $77.8^{\circ}$ correspond to the (111), (200), (220) and (311) crystal faces of Au nanoparticles. Similarly, the facecentered cubic silver crystals were found in the GH_Ag sample, with diffraction reflections at $38.4^{\circ}$ (111), $4 \overline{4} .6^{\circ}$ (200), $64.6^{\circ}(220)$ and $77.4^{\circ}(311)$. In the results obtained for the $\mathrm{GH} C u$ sample, relatively small but distinct reflection at $4 \overline{2} .6^{\circ}$ can be seen. It corresponds to the (111) planes of copper crystals. The other expected reflections at 50.8 and $74.4^{\circ}$ are not observed, most probably because of too low intensity. The average crystallite sizes calculated from the widening of the $\mathrm{Au}$ (111), $\mathrm{Ag}$ (111) and $\mathrm{Cu}(111)$ maxima by the Scherrer's formula were 61 $\mathrm{nm}, 84$ and $7 \mathrm{~nm}$, respectively. Intensity of XRD reflections observed for gold- and silver containing samples is high whereas this of $\mathrm{Cu}$ samples is low. This difference may be caused by higher atom scattering factors of gold and silver in comparison to copper as well as by highly nanocrystalline nature of copper particles. These results are in good agreement with UV-Vis spectra (Fig. 4) and SEM images, presented in Figure 6 .

As can be seen in Figure 6A and B, the obtained gold and silver nanoparticles are polydispersed, but with diameters not exceeding $100 \mathrm{~nm}$. The EDX analysis confirmed the presence of these metals at reasonable level, which was in accordance with metal-to-carbon ratio used in the reaction (Figure 6D). The SEM imaging of GH_Cu (Figure 6C) sample displayed very small copper particles. As can be seen in the inset, the size of nanoparticles do not exceed $10 \mathrm{~nm}$, which is almost
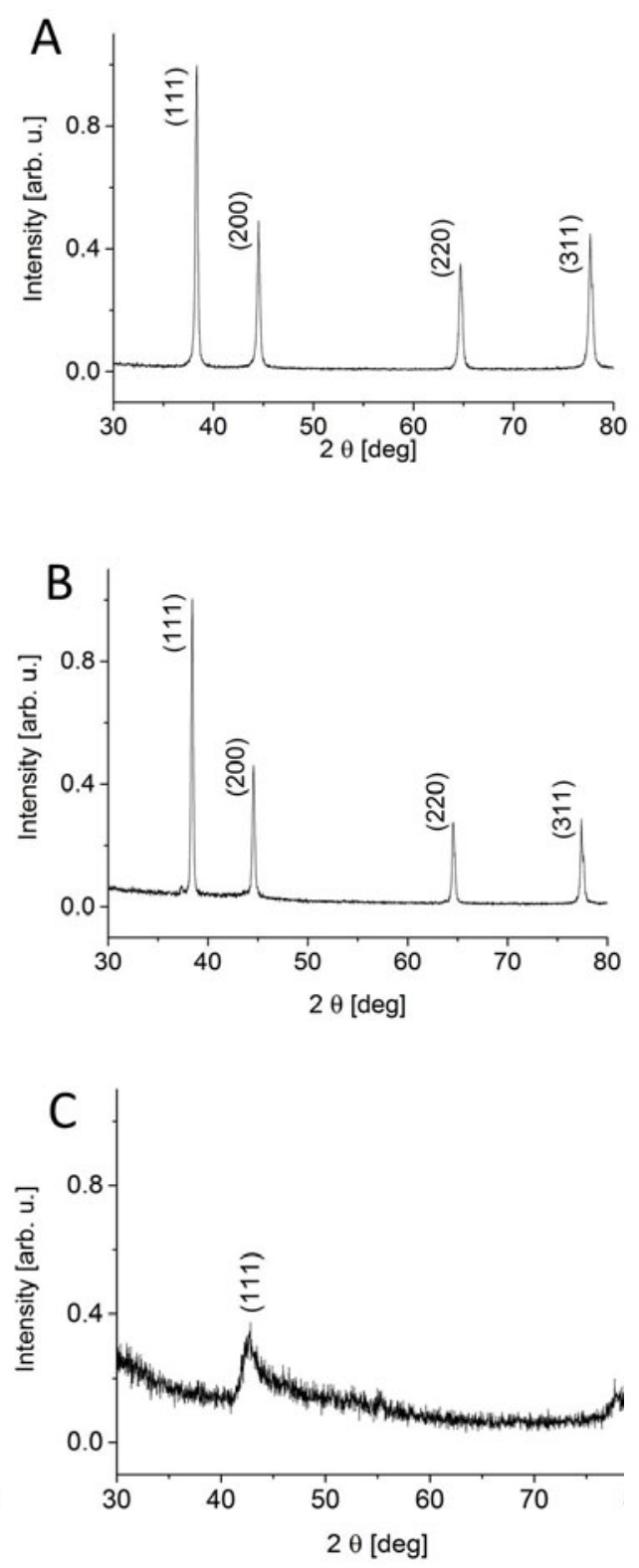

Figure 5. XRD patterns of A) $\left.\mathrm{GH}_{-} \mathrm{Au}, \mathrm{B}\right) \mathrm{GH} \_\mathrm{Ag}$ and C) CH_Cu samples

beyond the microscope resolution. The EDX analysis confirmed the presence of copper atoms (Figure $6 \mathrm{D}$ ). Taking into account the data obtained by UV-Vis and XRD spectroscopy, together with microscopic analysis it can be concluded, that $\mathrm{Cu}$ NPs below $10 \mathrm{~nm}$ were synthesized, which are much smaller as compared to other two metallic NPs, that is Ag and Au.

\section{STUDY ON THE CATALYTIC PERFORMANCE OF GRAPHENE HYDROGELS}

The catalytic activity of freshly prepared hydrogel/NPs composites was evaluated using model reaction of 4NP reduction. The change occurring in the solution was monitored visually as well as by UV-vis spectroscopy. Typical absorption band of 4-nitrophenol is observed at c.a. $320 \mathrm{~nm}$ and undergoes a bathochromic shift to 400 $\mathrm{nm}$, after adding an aqueous solution of $\mathrm{NaBH}_{4}$ due to the phenolate ion formation. For each sample the same amount of 4-NP solution was poured into the vials with different hydrogels. The solution of reductant was added directly before recording the UV-vis spectrum. In all cases 


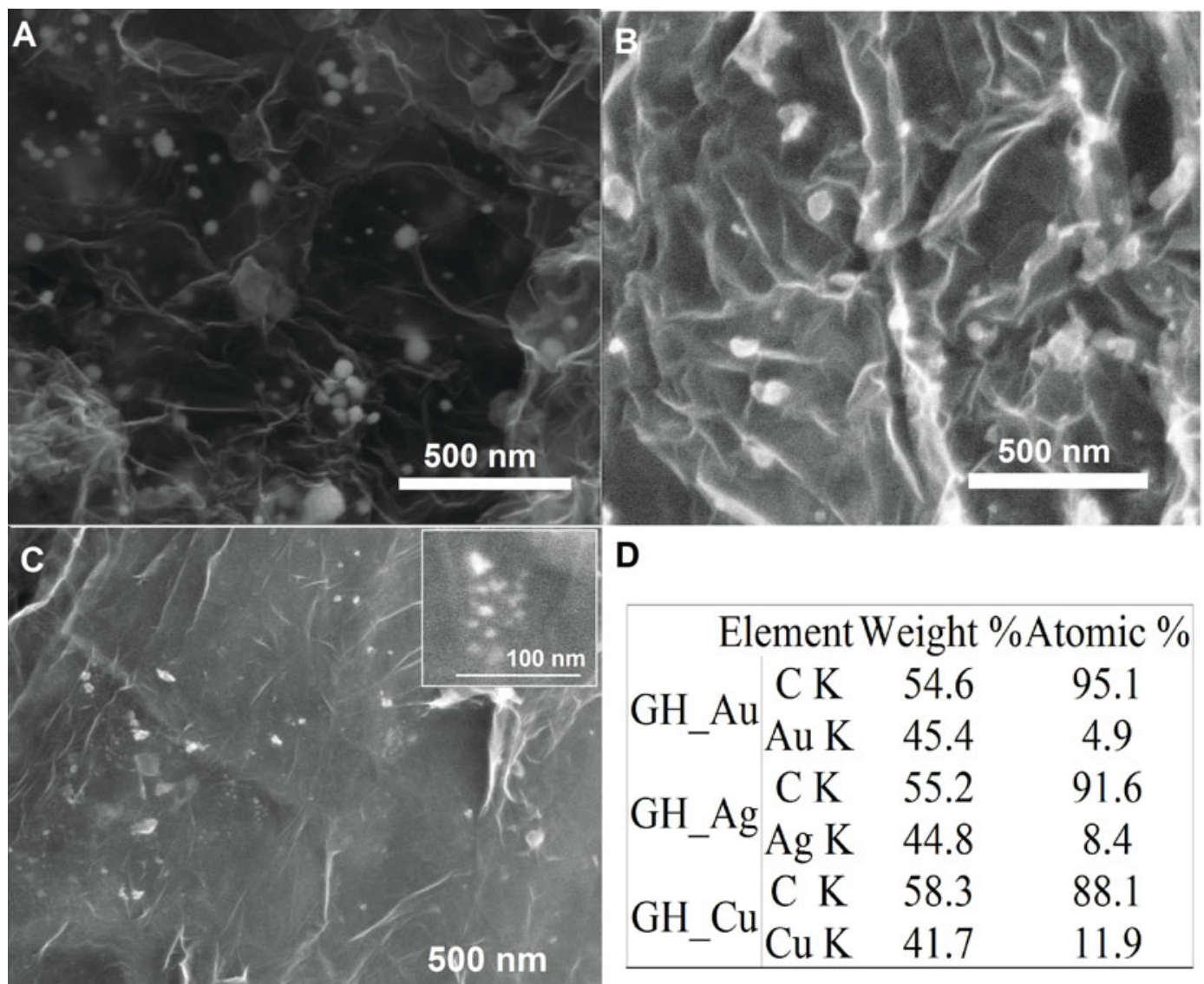

Figure 6. SEM images of A) $\mathrm{GH}_{-} \mathrm{Au}$; B) $\mathrm{GH} \_\mathrm{Ag}$ and C) $\mathrm{GH} \_\mathrm{Cu}$; D) table with elemental analysis of the samples

constant decrement of the $400 \mathrm{~nm}$ peak intensity was observed. In the case of GH_Au sample the appearance of new peak at c.a. $290 \mathrm{~nm}$ referring to 4-aminophenol can be seen (inset in Fig. 7A). Similarly, if GH_Ag sample was used as catalyst new peak at 290 and 230 $\mathrm{nm}$ were detected. Such observations are in agreement with the literature, proving the successful reduction of nitro to amino compound $\mathbf{d}^{\mathbf{3 0}-32}$.

It was also observed visually that the light yellow color of the 4-nitrophenol turned to yellowish green rapidly after adding the aqueous solution of $\mathrm{NaBH}_{4}$ (formation of 4-nitrophenolate ion) and faded with time to became almost colorless. The situation was different when GH_Cu hydrogel was implemented. In the case of GH_Cu sample, the disappearance of $400 \mathrm{~nm}$ peak was observed immediately after addition of the reducing agent. Neither absorption maximum due to 4-NP nor due to 4-AP was observed in the UV-vis spectrum (inset in Fig. 7C).

Figure 7 shows a linear correlation between $\ln (\mathrm{A})$ (A is the absorbance at $400 \mathrm{~nm}$ ) vs. reduction time, indicating that the reaction is a pseudo-first order for all three catalysts. However, in the case of $\mathrm{GH}_{-} \mathrm{Cu}$ it was not possible to determine the rate constant of the reaction as the reduction proceeded faster than the measurement time. For GH_Cu catalyst presented dependence was observed after 3 cycles of the reduction without regeneration of the catalyst (Fig. 7C). The rate constants $(\mathrm{k})$ determined from the slopes are $0.4 \cdot 10^{-3}$ $\mathrm{s}^{-1}, 0.7 \cdot 10^{-3} \mathrm{~s}^{-1}$ and $19.4 \cdot 10^{-3} \mathrm{~s}^{-1}$ for $\mathrm{GH} \_\mathrm{Au}, \mathrm{GH} \_\mathrm{Ag}$ and $\mathrm{GH} \_\mathrm{Cu}$, respectively. However, in the case of $\mathrm{GH}_{-} \mathrm{Cu}$ the calculated rate constant refers to the degradation of
4NP, not to its conversion to 4-aminophenol. According to the literature, the smaller the metallic NPs are, the higher catalytic activity they show ${ }^{2,3}$. Here, the average size obtained from XRD results is c.a $60 \mathrm{~nm}$ for $\mathrm{Au}$ NPs and $80 \mathrm{~nm}$ for Ag NPs, and from SEM images it can be seen, that the size distribution is wide. Thus, the observed catalytic activity is not as high as reported elsewhere ${ }^{21-23}$. In contrast, very small $\mathrm{Cu}$ NPs were obtained and they revealed outstanding properties. Such result is in particular beneficial, as the precursors for $\mathrm{Cu}$ NPs are freely available, more stable and less expensive as compared to silver or gold NPs precursors. One of the main problems with $\mathrm{Cu}$ NPs usage is their tendency to form oxides in the presence of air. The stability of the catalyst was monitored by XRD and UV-Vis spectroscopy and no changes were observed. The structure of graphene hydrogel/metallic NPs was not changed after the catalytic reaction. The metallic nanoparticles were present on the graphene sheets forming the hydrogel, as was controlled by SEM imaging. The leakage of metallic nanoparticles was also monitored by UV-Vis spectroscopy. As no plasmonic bands were present in the spectra after several cycles, it can be concluded, that no leakage occurred. Moreover no additional bands, for example due to metallic oxide formation were observed after weeks. The XRD pattern showed, that the metallic state was kept after the catalytic run for all analyzed samples, including $\mathrm{Cu}$ containing composite. In order to additionally verify the catalytic performance of $\mathrm{GH}_{-} \mathrm{Cu}$, the reduction of methylene blue (MB) with $\mathrm{NaBH}_{4}$ in the presence of $\mathrm{GH}_{-} \mathrm{Cu}$ was carried out. The $\mathrm{MB}$ is a water soluble dye used in medicine and pharmacy, 

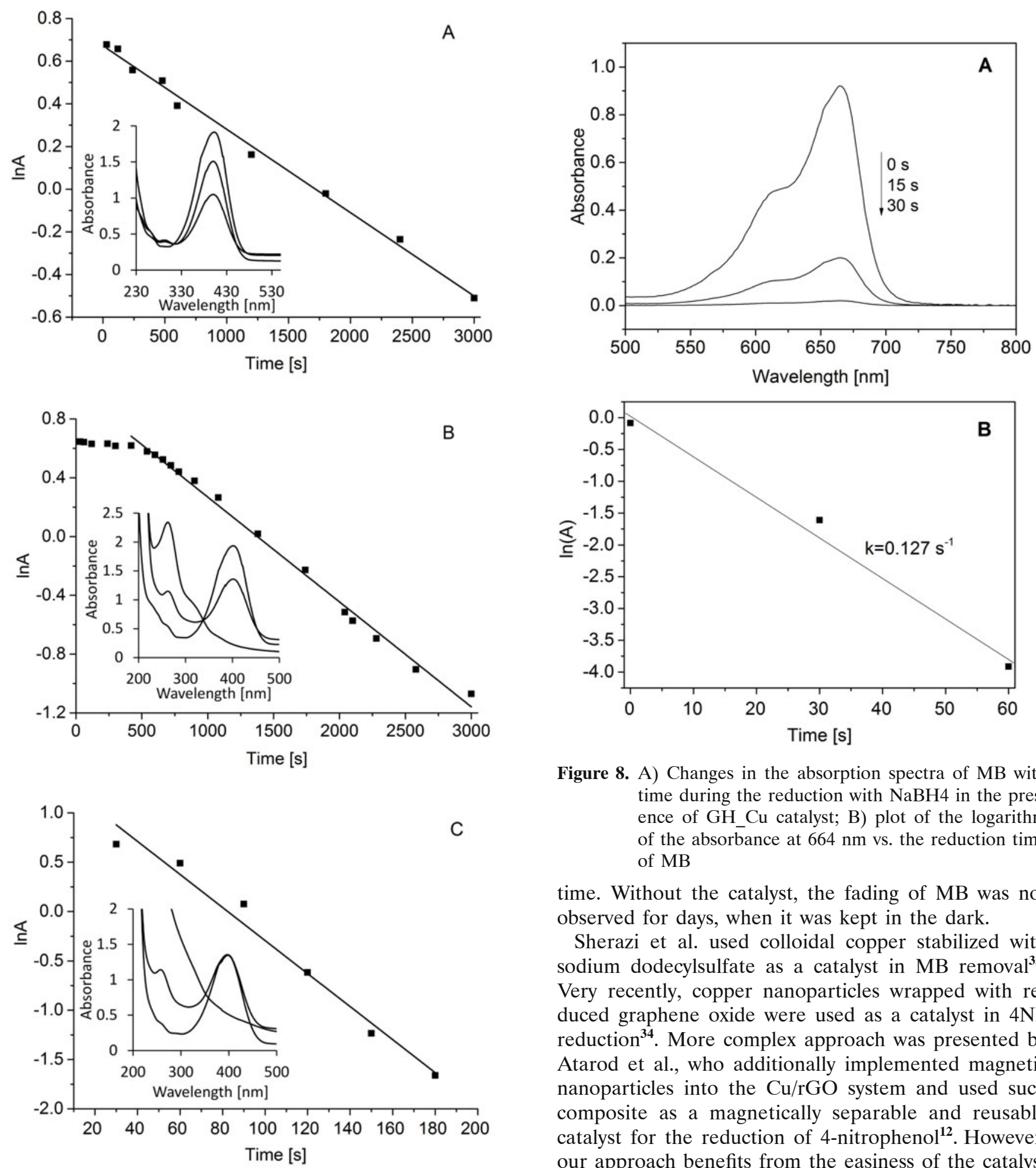

Figure 7. Plot of the logarithm of the absorbance at $400 \mathrm{~nm}$ vs. the reduction time for A) $\mathrm{GH}_{-} \mathrm{Au}$; B) $\mathrm{GH} \_\mathrm{Ag}$ and C) $\mathrm{GH}_{-} \mathrm{Cu}$. Insets present changes in the absorption spectra of 4NP with time

causing however cardiovascular, dermatological and hematological problems. The methods of its removal from wastewaters are sought. The reaction progress was monitored by UV-Vis spectroscopy. The obtained results are presented in Figure 8. The decolorization of methylene blue solution occurred immediately after addition of reductant, when catalyst was present. The rate constant determined from the slope was equal to $0.127 \mathrm{~s}^{-1}$. The reaction was repeated three times without catalyst regeneration, showing no increase of the reaction

Figure 8. A) Changes in the absorption spectra of $\mathrm{MB}$ with time during the reduction with $\mathrm{NaBH} 4$ in the presence of GH_Cu catalyst; B) plot of the logarithm of the absorbance at $664 \mathrm{~nm}$ vs. the reduction time of $\mathrm{MB}$

time. Without the catalyst, the fading of MB was not observed for days, when it was kept in the dark.

Sherazi et al. used colloidal copper stabilized with sodium dodecylsulfate as a catalyst in MB removal ${ }^{33}$. Very recently, copper nanoparticles wrapped with reduced graphene oxide were used as a catalyst in 4NP reduction $^{\mathbf{3 4}}$. More complex approach was presented by Atarod et al., who additionally implemented magnetic nanoparticles into the $\mathrm{Cu} / \mathrm{rGO}$ system and used such composite as a magnetically separable and reusable catalyst for the reduction of 4-nitrophenol ${ }^{12}$. However, our approach benefits from the easiness of the catalyst preparation, removal from the reaction mixture and regeneration.

\section{CONCLUSIONS}

Simple method of graphene hydrogels with embedded metal nanoparticles was presented. Graphene/Cu NPs hydrogels were found to be the most active towards degradation of two model compounds, that is 4-nitrophenol and methylene blue dye. The reaction was completed within few seconds. In the case of $\mathrm{Au}$ and Ag NPs supported on graphene hydrogel the reduction of 4-NP lead to the 4-aminophenol formation, with satisfactory rate constants. High adsorption ability of graphene and effective electron transfer from graphene to metallic 
nanoparticles make the proposed system very efficient in the chemical reduction of different compounds. The usage of graphene hydrogel/metallic NPs as a reusable heterogeneous catalyst in various reaction will be furthered studied.

\section{LITERATURE CITED}

1. Sheldon, R.A. \& van Bekkum, H. (Eds.) Fine Chemicals through Heterogeneous Catalysis. Wiley-VCH 2001.

2. Mitsudomea, T. \& Kaneda, K. (2013). Gold nanoparticle catalysts for selective hydrogenations. Green Chem. 15, 2636-2654. DOI: 10.1039/C3GC41360H.

3. Zhao, P., Feng, X., Huang, D., Yang, G. \& Astruc, D. (2015) Basic concepts and recent advances in nitrophenol reduction by gold - and other transition metal nanoparticles. Coord. Chem. Rev. 287, 114-136. DOI:10.1016/j.ccr.2015.01.002.

4. Santos, K. de O., Elias, W.C., Signori, A.M., Giacomelli, F.C., Yang, H. \& Domingos, J.B. (2012). Synthesis and Catalytic Properties of Silver Nanoparticle-Linear Polyethylene Imine Colloidal Systems. J. Phys. Chem. C 116, 4594-4604. DOI: 10.1021/jp2087169.

5. Gao, S., Zhang, Z., Liu, K. \& Dong, B. (2016). Direct evidence of plasmonic enhancement on catalytic reduction of 4-nitrophenol over silver nanoparticles supported on flexible fibrous networks. Appl. Catal. B: Environ 188, 245-252. DOI: 10.1016/j.apcatb.2016.01.074.

6. Nasrollahzadeh, M., Sajadi, S.M., Rostami-Vartooni, A., Bagherzadeh, M. \& Safari, R. (2015). Immobilization of copper nanoparticles on perlite: Green synthesis, characterization and catalytic activity on aqueous reduction of 4-nitrophenol. J. Mol. Cat. A Chem. 400, 22-30. DOI: 10.1016/j.molcata.2015.01.032.

7. Hatamifard, A., Nasrollahzadeh, M. \& Lipkowski J. (2015). Green synthesis of a natrolite zeolite/palladium nanocomposite and its application as a reusable catalyst for the reduction of organic dyes in a very short time. RSC Adv. 5, 91372-91381. DOI: 10.1039/C5RA18476B.

8. Rostami-Vartooni, A., Nasrollahzadeh, M. \& Alizadeh, M. (2016). Green synthesis of seashell supported silver nanoparticles using Bunium persicum seeds extract: Application of the particles for catalytic reduction of organic dyes. J. Coll. Interf. Sci. 470, 268-275. DOI: 10.1016/j.jcis.2016.02.060.

9. Tajbakhsh, M., Alinezhad, H., Nasrollahzadeh, M. \& Kamali, T.A. (2016). Green synthesis of the Ag/HZSM-5 nanocomposite by using Euphorbia heterophylla leaf extract: A recoverable catalyst for reduction of organic dyes. J. Alloy. Compd. 685, 258-265. DOI: 10.1016/j.jallcom.2016.05.278.

10. Rostami-Vartooni, A., Nasrollahzadeh, M. \& Alizadeh, M. (2016). Green synthesis of perlite supported silver nanoparticles using Hamamelis virginiana leaf extract and investigation of its catalytic activity for the reduction of 4-nitrophenol and Congo Eds. J. Alloy. Compd. 680, 309-314. DOI: 10.1016/j. jallcom.2016.04.008.

11. Nasrollahzadeh, M., Atarod, M., Jaleh, B. \& Gandomirouzbahani, M. (2016). In situ green synthesis of Ag nanoparticles on graphene oxide/ $\mathrm{TiO}_{2}$ nanocomposite and their catalytic activity for the reduction of 4-nitrophenol, congo red and methylene blue. Ceram. Inter. 42, 8587-8596. DOI: 10.1016/j. ceramint.2016.02.088.

12. Atarod, M., Nasrollahzadeh, M. \& Sajadi, S.M. (2015). Green synthesis of a $\mathrm{Cu} /$ reduced graphene oxide $/ \mathrm{Fe}_{3} \mathrm{O}_{4}$ nanocomposite using Euphorbia wallichii leaf extract and its application as a recyclable and heterogeneous catalyst for the reduction of 4-nitrophenol and rhodamine B. RSC Adv. 5, 91532-91543. DOI: 10.1039/c5ra17269a.

13. Fakhri, P., Nasrollahzadeh, M. \& Jaleh, B. (2014). Graphene oxide supported Au nanoparticles as an efficient catalyst for reduction of nitro compounds and Suzuki-Miyaura coupling in water. RSC Adv. 4, 48691-48697. DOI: 10.1039/C4RA06562J.
14. Nasrollahzadeh, M., Sajadi, S.M., Rostami-Vartooni, A., Alizadeh, M. \& Bagherzadeh, M. (2016). Green synthesis of the Pd nanoparticles supported on reduced graphene oxide using barberry fruit extract and its application as a recyclable and heterogeneous catalyst for the reduction of nitroarenes. J. Col. Interf. Sci. 466, 360-368. DOI: 10.1016/j.jcis.2015.12.036.

15. Atarod, M., Nasrollahzadeh, M. \& Sajadi, S.M. (2016). Green synthesis of $\mathrm{Pd} / \mathrm{RGO} / \mathrm{Fe}_{3} \mathrm{O}_{4}$ nanocomposite using Withania coagulans leaf extract and its application as magnetically separable and reusable catalyst for the reduction of 4-nitrophenol. J. Col. Interf. Sci. 465, 249-258. DOI: 10.1016/j. jcis.2015.11.060.

16. Navalon, S., Dhakshinamoorthy, A., Alvaro, M. \& Garcia, H. (2016). Metal nanoparticles supported on two-dimensional graphenes as heterogeneous catalysts. Coord. Chem. Rev. 312, 99-148. DOI: 10.1016/j.ccr.2015.12.005.

17. Julkapli, N.M. \& Bagheri, S. (2015). Graphene supported heterogeneous catalysts: An overview. Int. J. Hydr. Energ. 40, 948-979.

18. Xu, Y., Sheng, K., Li, Ch. \& Shi, G. (2010). Self-Assembled Graphene Hydrogel via a One-Step Hydrothermal Process. ACS Nano 4(7), 4324-4330. DOI: 10.1021/nn101187z.

19. Xia, X.H., Chao, D.L., Zhang, Y.Q., Shen, Z.X. \& Fan, H.J. (2015). Three-dimensional graphene and their integrated electrodes. Nano Today 9(6), 785-807. DOI: 10.1016/j. nantod.2014.12.001.

20. Fang, Q., Shen, Y. \& Chen, B. (2015). Synthesis, decoration and properties of three-dimensional graphene-based macrostructures: A review. Chem. Engine. J. 264, 753-771. DOI: 10.1016/j.cej.2014.12.001.

21. Li, J., Liu, Ch-Y. \& Liu, Y. (2012). Au/graphene hydrogel: synthesis, characterization and its use for catalytic reduction of 4-nitrophenol. J. Mater. Chem. 22, 8426-8430. DOI: 10.1039/ C2JM16386A.

22. Dubey, S.P., Dwivedi, A.D., Kim, I-Ch, Sillanpa, M., Kwon, Y-N. \& Lee, Ch. (2014). Synthesis of graphene-carbon sphere hybrid aerogel with silver nanoparticles and its catalytic and adsorption applications. Chem. Engine. J. 244, 160-167. DOI: 10.1016/j.cej.2014.01.042.

23. He, Y., Zhang, N., Gong, Q., Li, Z., Gao, J. \& Qiu, H. (2012). Metal nanoparticles supported graphene oxide 3D porous monoliths and their excellent catalytic activity. Mater. Chem. Phys. 134, 585-589. DOI:10.1016/j.matchemphys.2012.04.011.

24. Wu, T., Chen, M., Zhang, L., Xu, X., Liu, Y., Yan, J., Wang, W. \& Gao, J. (2013). Three-dimensional graphene-based aerogels prepared by a self-assembly process and its excellent catalytic and absorbing performance. J. Mater. Chem. A 1, 7612-7621. DOI: 10.1039/C3TA10989E.

25. ICDD PDF-2 Database Release 1998, ISSN 1084-3116.

26. Kondratowicz, I., Żelechowska, K. \& Sadowski, W. (2015). Optimization of graphene oxide synthesis and its reduction. In O. Fesenko \& L. Yatsenko (Eds.), Nanoplasmonics, NanoOptics, Nanocomposites, and Surface Studies (pp. 467-484). Springer.

27. Kondratowicz, I. (2014). Porous graphene electrodes. Synthesis, modifications and characterization. Unpublished Master Thesis. Gdansk University of Technology, Gdansk, Poland.

28. Yang, D., Velamakanni, A., Bozoklu, G., Park, S., Stoller, M., Piner, R.D., Stankovich, S., Jung, I., Field, D.A., Ventrice C.A. \& Ruoff, R.S. (2009). Chemical analysis of graphene oxide films after heat and chemical treatments by X-ray photoelectron and Micro-Raman spectroscopy. Carbon 47, 145-152. DOI: 10.1016/j.carbon.2008.09.045.

29. Lisiecki, I. \& Pileni, M.P. (1995). Copper Metallic Particles Synthesized "in Situ" in Reverse Micelles: Influence of Various Parameters on the Size of the Particles. J. Phys. Chem. 99, 5077-5082. DOI: 10.1021/j100014a030.

30. Wunder, S., Polzer, F., Lu, Y. \& Mei, Y. (2010). Ballauff, M. Kinetic Analysis of Catalytic Reduction of 4-Nitrophenol 
by Metallic Nanoparticles Immobilized in Spherical Polyelectrolyte Brushes. J. Phys. Chem. C. 114, 8814-8820. DOI: 10.1021/jp101125j.

31. Wunder, S., Lu, Y., Albrecht, M. \& Ballauff, M. (2011). Catalytic Activity of Faceted Gold Nanoparticles Studied by a Model Reaction: Evidence for Substrate-induced Surface Restructuring. ACS Catal. 1, 908-916. DOI: 10.1021/cs200208a.

32. Gu, S., Wunder, S., Lu, Y. \& Ballauff, M. (2014). Kinetic Analysis of the Catalytic Reduction of 4-Nitrophenol by Metallic Nanoparticles. J. Phys. Chem. C 118, 18618-18625. DOI: $10.1021 / \mathrm{jp} 5060606$.

33. Sherazi, S.T.H., Soomro, R.A., Uddin, S. \& Memon, N. (2014). Synthesis and characterizations of highly efficient copper nanoparticles and their use in ultrafast catalytic degradation of organic dyes. Adv. Mater. Res. 829, 93-99. DOI: 10.4028/ www.scientific.net/AMR.829.93.

34. Hang, L., Zhao, Y., Zhang, H., Liu, G., Cai, W., Li, Y. \& Qu, L. (2016). Copper nanoparticle@graphene composite arrays and their enhanced catalytic performance. Acta Mater. 105, 59-67. DOI:10.1016/j.actamat.2015.12.029. 